\title{
Assessment of the Low-Cycle Strain-Induced Martensite Transformation in AISI 316 Stainless Steel by Magnetic and Acoustic Nondestructive Methods
}

\author{
C. S. Kim
}

Department of Materials Science and Engineering, Chosun University, Gwangju, Republic of Korea chs2865@chosun.ac.kr

The strain-induced martensite transformation during the low-cycle fatigue in austenitic AISI 316 stainless steel was investigated by magnetic and acoustic nondestructive methods. The low-cycle fatigue test was performed at various strain amplitudes. The volume fraction of $\alpha^{\prime}$-martensite was determined for the fatigue-failed specimens by magnetic property measurements with further microstructure detection. The cyclic hardening behavior was discussed in terms of the $\alpha^{\prime}$-martensite transformation. The volume fraction of $\alpha^{\prime}$-martensite was growing with the strain amplitude. An increase in the $\alpha^{\prime}$-martensite fraction was evaluated with the nonlinear ultrasonic parameter. The $\alpha^{\prime}$-martensite fraction may distort the lattice in austenitic stainless steel, resulting in the distortion of an ultrasonic wave. From this distortion, superharmonics may be generated with the $\alpha^{\prime}$-martensite nucleation, which strongly depends on the strain amplitude. The relationship between nonlinear acoustic characteristic and the volume fraction of $\alpha^{\prime}$-martensite is linear.

Keywords: strain-induced martensite, low-cycle fatigue, acoustic nonlinearity, stainless steel, super-harmonics.

Introduction. Austenitic AISI 316 steels are widely used in the chemical and energy industry components due to their good corrosion resistance, weldability, and excellent mechanical properties. These advantages are critical under severe working conditions such as high temperature, high pressure, cyclic loading, etc. There is a growing interest in the failure prediction of austenitic stainless steels: structural components designed for high-temperature and high-pressure environments of power and chemical plans strongly deteriorate during their operation, which raise safety and integrity concerns. In addition, desirable or undesirable unforeseen states may occur during service, which may influence the material state or behavior. Changes to the material properties can never be detected in facilities during operation using conventional destructive methods. Therefore, the integrity assurance and life prediction of structural components is necessary for the structural safety and reliable operation [1]. For the life prediction and assurance of mechanical structures, the precise nondestructive testing for fatigue-damaged materials is critical.

An AISI 316 steel has a paramagnetic and metastable phase, which is easily transformed into the strain-induced martensite, $\alpha^{\prime}$-phase [2]. This strain-induced martensite fraction is in the ferromagnetic phase. The magnetic property is very sensitive to any microstructural changes in the materials. Therefore, magnetic methods have been applied to various steels to estimate the microstructural changes, such as phase transformation and variation of grain and particle sizes [3]. The magnetic properties of steels (i.e., ferromagnetic materials) are known to depend on their microstructural characteristics, such as composition, dislocation, size of grains and precipitates, etc. Nonlinear ultrasonic analysis can promote an understanding of the evolution and accumulation of plastic deformation of materials at very early stages of fatigue. The enhanced generation of high-order harmonics due to the distortion of ultrasonic waves propagated through the materials has been reported by numerous authors, and recent experimental studies and new 
physical models provide a strong evidence that the nonlinear ultrasonic parameter $\beta$ is highly dependent on the fatigue degradation [4].

The aim of the present study is to investigate the low-cycle strain-induced degradation of AISI 316 stainless steels using magnetic and acoustic methods. The nonlinear ultrasonic measurement is performed in austenitic stainless steels and the oblique incidence technique has been applied to obtain the nonlinear ultrasonic parameter. Also, the magnetic characteristics are measured for the microstructural evolution; various metallurgical observations are made after the fatigue failure at various strain amplitudes. An attempt is made to establish the microstructure effect on the generation of high-order harmonics of the ultrasonic wave and variation in the magnetic properties during the cyclic loading in austenitic stainless steels by correlating the results of nondestructive evaluation (NDE) characteristics with the microstructural evolution data.

1. Experimental Procedure. Flat specimens of $15 \mathrm{~mm}$ gauge length were produced from from hot-rolled AISI 316 stainless steel specimen and subjected to the low-cycle fatigue (LCF) tests at room temperature in compliance with the ASTM E 606 standard test method regulations. The total strain amplitude was controlled under fully reversed tension and compression with plates. The total strain amplitude was controlled through the range from 0.5 to $1.7 \%$ with $3 \cdot 10^{-3} \mathrm{~s}^{-1}$ strain rate. The magnetic characteristics of fatigue damaged specimens have been evaluated to investigate the phase transformation such as strain-induced martensite transformation, since this martensite fraction is the ferromagnetic phase. The magnetic characteristics such as the coercivity $\left(H_{c}\right)$, remanance $\left(M_{r}\right)$, and saturation magnetization $\left(M_{S}\right)$ were obtained from the magnetic induction $(B)$ and magnetic field $(H)$ curve, magnetic hysteresis through the vibrating sample magnetometer (VSM) measurement system.

A high-power pulse generator (Ritec, RAM5000 SNAP) was used to provide high-power tone-burst signals to the transmitter. A schematic diagram of the experimental setup for measuring the acoustic nonlinearity of an oblique incidence measurement technique is shown in Fig. 1. The oblique incidence technique has a practical utility in structural facilities of plants due to its easy accessibility to the surfaces of structures under study. The received signal was digitally processed using the fast Fourier transform (FFT) power spectral analysis to obtain amplitudes of the fundamental and second-order harmonic frequencies. The amplitude of the fundamental frequency $\left(A_{1}\right)$ was determined from the FFT of the signal fed to channel 1 ; then the nonlinear ultrasonic parameter $(\beta)$ was measured using $\beta \propto A_{2} / A_{1}^{2}$, which is the relative value $\beta^{\prime}$ of the absolute parameter $\beta$ $[5]$.

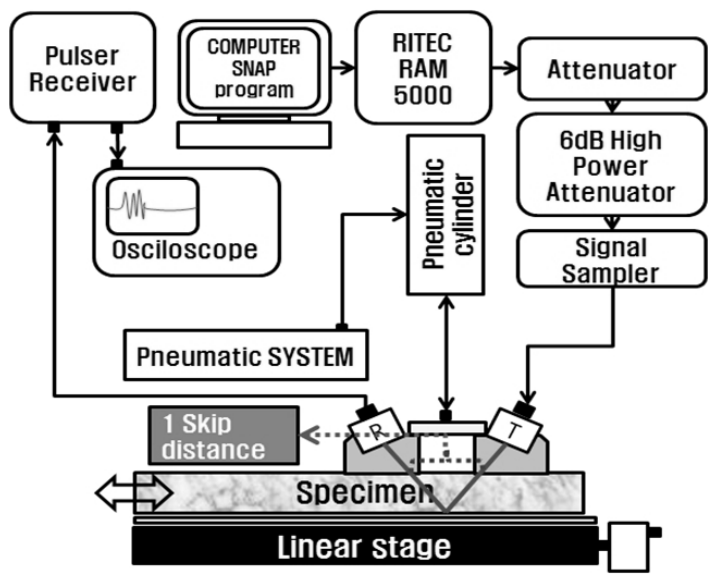

Fig. 1. Schematic diagram of the experimental setup for measuring the acoustic nonlinearity by the oblique incidence technique ( $R$ and $T$ are receiver and transmitter probes, respectively). 
2. Results and Discussion. Figure 2 depicts the low-cycle fatigue results of austenitic AISI 316 stainless steel. The stress amplitude was varied as a function of the number of cycles with the total stain amplitude. The number of cycles to fatigue fracture decreased with strain amplitude. This allowed for examination of variation in the maximum load with the number of cycles, which could indicate whether the material was cyclically hardened, softened, or stable.

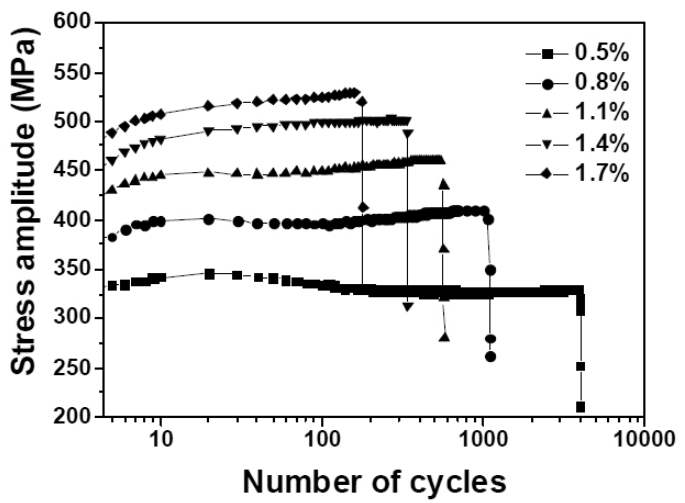

Fig. 2. Variation of stress amplitude as a function of number of fatigue cycles at various strain amplitudes featuring two cyclic hardening patterns.

The stress amplitude increased within several tens of cycles, which corresponded to the first cyclic hardening, and then a slight softening was observed at $0.5 \%$ strain amplitude. From 0.8 to $1.1 \%$ of the strain amplitude, the second hardening was observed. Over the stain amplitude of $1.1 \%$, there is no saturation phenomenon of stress amplitude and second hardening. For the first hardening, the dislocation multiplication and increase of dislocation density may be the primary reason of hardening. The subsequent second hardening was caused by the strain-induced $\alpha^{\prime}$-martensite transformation with fatigue deformation. The surface microstructures of the austenitic 316 stainless steel corresponding strain amplitude of $1.7 \%$ are shown in Fig. $3 a$ and $3 b$, and they are compared with those of the as-received specimen.

The as-received specimen consisted of a singular $\gamma$-austenitic phase. However, the dual phases such as $\gamma$-austenite and $\alpha^{\prime}$-martensite have been observed in fatigue-damaged specimens. Figure $3 \mathrm{c}$ and $3 \mathrm{~d}$ shows transmission electron micrographs of the dislocation substructure in the as-received and fatigue failure at strain amplitude of $1.7 \%$. The typical dislocation cell microstructure was well-developed. The first hardening within several tens of cycles was caused by the dislocation multiplication and increased dislocation density, and then the cyclic softening was attributed to this dislocation cell formation by fatigue.

Figure 4 depicts the variation in the magnetic properties of fatigue damaged specimens. The magnetic parameters such as $H_{c}, M_{s}$ and $M_{r}$ increased as a function of strain amplitude. The monotonic increase in the magnetic parameters with the strain amplitude could be closely related to magnetic particles in austenitic AISI 316 stainless steels. As mentioned earlier, the austenite matrix is in the non-ferromagnetic phase. In addition, there is another reason for the increase in the magnetic parameters in this austenitic AISI 316 stainless steel. The martensite fraction effect can be an important factor of ferromagnetic phase that may affect the magnetic properties.

The strain-induced martensite could have a reasonable influence on the magnetic property. The slip and twin boundaries could present an obstacle to the magnetic domain movement, resulting in an increase in the magnetic parameters. In addition, dislocations may be one of primary effects on the domain wall movement, which is a reasonable cause of the variation in magnetic parameters. Most of all, the strain-induced martensite had a 

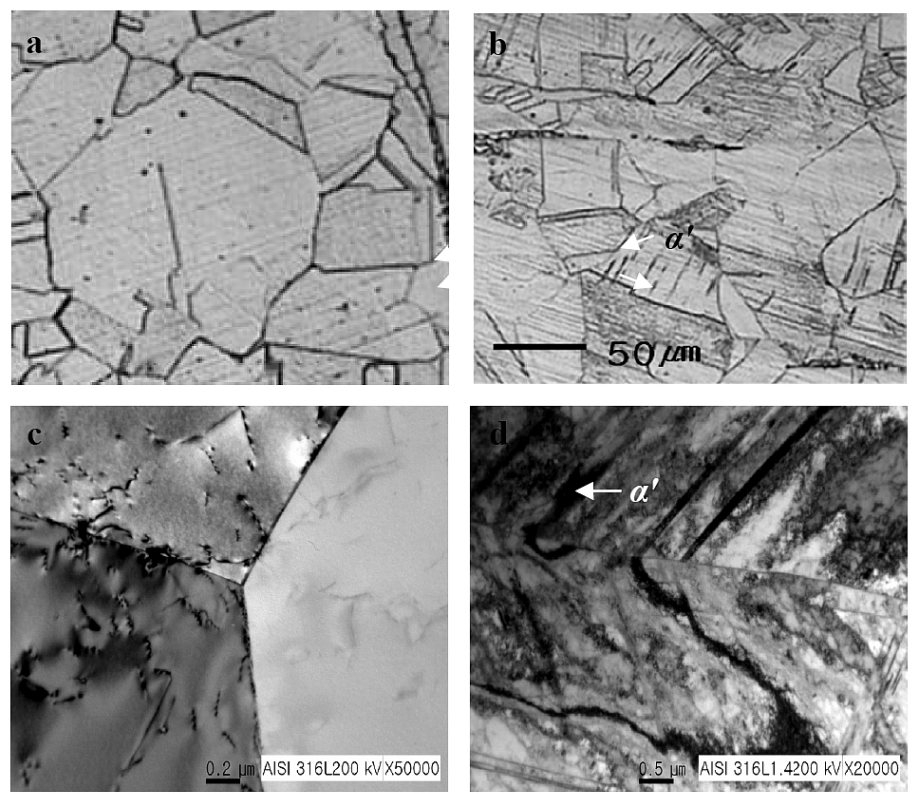

Fig. 3. Optical and transmission electron micrographs showing $\alpha^{\prime}$-martensite and dislocation substructure as indicated by arrows: (a) optical image of as-received sample showing deformation free and many annealing twins; (b) optical image of $1.7 \%$ strain amplitude specimen showing $\alpha^{\prime}$-martensite phase; (c) TEM image of as-received specimen showing rare dislocation density; (d) TEM image of $1.7 \%$ strain amplitude specimen showing $\alpha^{\prime}$-martensite and high dislocation density. (The number of cycles up to failure, $N_{f}=182$.)

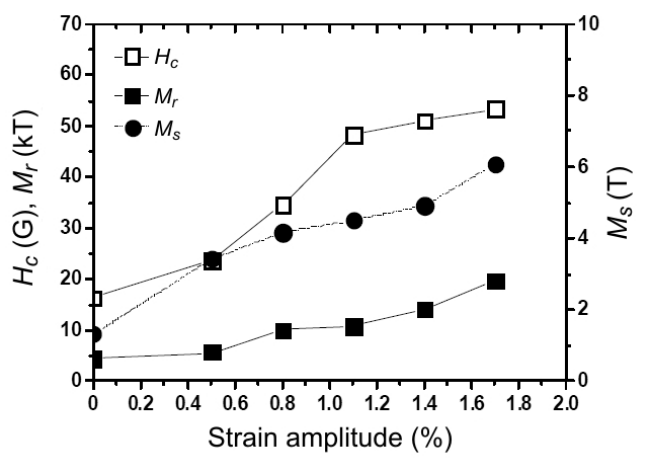

Fig. 4. Change in the coercivity $\left(H_{c}\right)$, magnetization $\left(M_{s}\right)$, and remanance $\left(M_{r}\right)$ of the fatiguedamaged specimens with different strain amplitudes.

reasonable influence on the increase in magnetic properties because of its ferromagnetic property. The austenite is a typical paramagnetic phase in steel microstructure. However, the strain-induced martensite ( $\alpha^{\prime}$ phase) is primarily ferromagnetic phase. Therefore, the magnetic parameters of fatigue-damaged specimens are originally caused by the $\alpha^{\prime}$ phase during the fatigue damage. Figure 5 shows the acoustic signal and its spectrum of the ultrasonic wave. In this test, a $5 \mathrm{MHz}$ transducer was used as a transmitter and a $10 \mathrm{MHz}$ transducer was used as a receiver. Because a narrow band signal is advantageous for effectively detecting the higher harmonic amplitude, a $5 \mathrm{MHz}$ tone-burst signal with a "window" was used to transmit the waveform. The length of the burst was 10 cycles, which confirmed that both the fundamental frequency and its double frequency for the second-order harmonic wave were detected and identified quite accurately. 

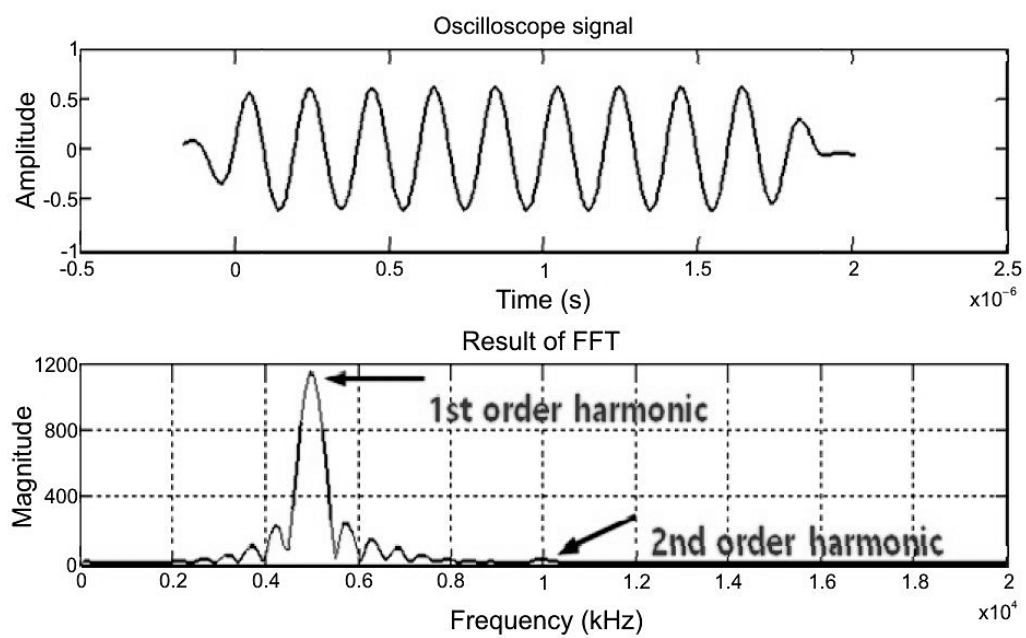

Fig. 5. Ultrasonic signal wave and its spectral waveform for $1.7 \%$ strain amplitude showing 1 st and 2nd order ultrasonic harmonics.

The second-order harmonic amplitude depends on the fundamental frequency amplitude. From the theoretical background, the second-order harmonic amplitude would be linearly proportional to the square of the fundamental amplitude, which implies that parameter $\beta^{\prime}$ is constant regardless of the variation in the fundamental frequency amplitude when the wave number and propagation distance are fixed. Figure 6 depicts the variation in the acoustic nonlinearity of AISI 316 stainless steel fatigue-failed specimens at various strain amplitudes. The acoustic nonlinearity increased with strain amplitudes due to the fatigue-driven plastic deformation, and it is closely related to the strain amplitudes and fatigue deformation accumulation. To reveal the microstructural features that influence the distortion of the acoustic wave, resulting in super-harmonic generation, we consider the microstructural evolution during the fatigue damage process. It has been reported by many authors that during cyclic loading, dislocations play an important role in generating ultrasonic wave super-harmonics. The nonlinear acoustic parameter is related to the dislocation loop length $(L)$, dislocation density $(\Delta)$, and stress $(\sigma)$ via the following equation [6]:

$$
\beta \propto \Delta L^{4} \sigma
$$

Equation (1) shows that $\beta$ is linearly dependent on the stress and dislocation density. Here the variation in the acoustic nonlinearity parameter with the strain amplitude has been discussed with consideration for the effect of both parameter strain and microstructural changes during fatigue. In TEM microstructure, as shown in Fig. 3, increased dislocation densities were observed. Clear dislocation cell structures have been observed in the TEM microstructure. The cell interior is dislocation-free and corresponds to fatigue strain softening. Martensite is a distorted bcc ferrite lattice in parts of a steel crystal that still contains the dissolved carbon and is full of defects. The share of atomically dissolved carbon in the martensite exceeds the value acceptable for a stable ferrite, distorting the lattice. This lattice distortion can be the primary reason for the wave distortion when the ultrasonic wave propagates through the test materials, since super-harmonics may be generated with a nucleation of strain-induced martensite, which strongly depends on the strain amplitude. Noteworthy is that the NDE technique allows one to use the acoustic nonlinearity parameter for characterizing the $\alpha^{\prime}$-martensite during fatigue strain-induced deformation of austenitic stainless steels. 


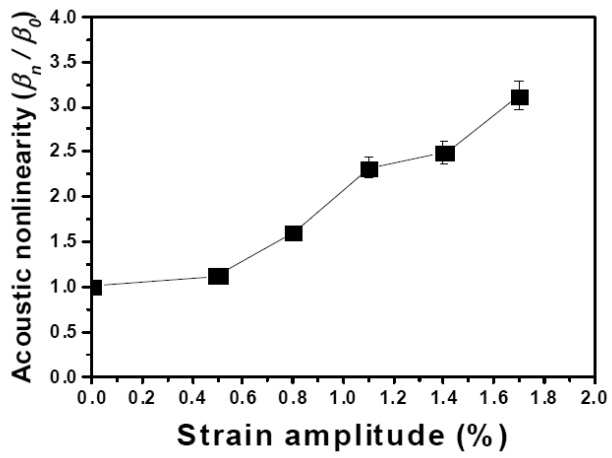

Fig. 6. Variation in the acoustic nonlinearity of AISI 316 stainless steel fatigue-failed specimens under various strain amplitudes $\left(\beta_{0}\right.$ is a nonlinearity parameter of the as-received specimen and $\beta_{n}$ is a nonlinearity parameter of the fatigue-failed specimen).

Conclusions. The martensite transformation ( $\alpha^{\prime}$ phase) subjected to low-cycle fatigue damage in AISI 316 steel was evaluated with the magnetic and acoustic nondestructive evaluation techniques. The $\alpha^{\prime}$ phase (strain-induced martensite) has been generated and the volume fraction increased with cyclic stain amplitude. The increase in the $\alpha^{\prime}$-martensite fraction was evaluated using a nonlinear ultrasonic parameter. The $\alpha^{\prime}$-martensite may distort the lattice in austenitic stainless steels, resulting in the ultrasonic wave distortion. The superharmonics were generated with a nucleation of the strain-induced martensite and exhibited their strong dependence on the strain amplitude. This study results strongly suggest that the acoustic nonlinearity parameter and magnetic coercivity can be successfully applied to characterize the $\alpha^{\prime}$-martensite during cyclic deformation of austenitic stainless steel.

Acknowledgments. This research was supported by Basic Science Research Program through the National Research Foundation of Korea (NRF) funded by the Ministry of Education (2017R1D1A3B03028681).

1. C. W. Ziemian, R. D. Ziemian, and K. V. Haile, "Characterization of stiffness degradation caused by fatigue damage of additive manufactured parts," Mater. Design, 109, 209-218 (2016).

2. H. J. Leber, M. Niffenegger, and B. Tirbonod, "Microstructural aspects of low cycle fatigued austenitic stainless tube and pipe steels," Mater. Charact., 58, No. 10, 1006-1015 (2007).

3. K. S. Ryu, C. S. Kim, U. B. Baek, and J. S. Lee, "Nondestructive evaluation for remanent life of aged $12 \mathrm{Cr}$ ferrite heat resisting steel by reversible permeability," $J$. Magn. Magn. Mater., 326, 257-260 (2013).

4. M. A. Drewry and P. D. Wilcox, "One-dimensional time-domain finite-element modelling of nonlinear wave propagation for non-destructive evaluation," $N D T \& E$ Int., 61, 45-52 (2014).

5. A. Viswanath, B. P. C. Rao, S. Mahadevan, et al., "Nondestructive assessment of tensile properties of cold worked AISI type 304 stainless steel using nonlinear ultrasonic technique," J. Mater. Process. Tech., 211, No. 3, 538-544 (2011).

6. Y. Q. Cai, J. Z. Sun, C. J. Liu, et al., "Relationship between dislocation density in P91 steel and its nonlinear ultrasonic parameter," J. Iron Steel Res. Int., 22, 1024-1030 (2015). 